\title{
PAK up your troubles
}

PAKs (p21-activated kinases) are key regulators of the actin cytoskeleton that, in neurons, have a role in dendritic spine morphogenesis. In mice, mutation of Pak3 causes X-linked, non-specific mental retardation, and perturbation of the PAK pathway leads to cognitive deficits and dendritic spine defects. Now, in Nature Neuroscience, Zhao et al. report a loss of PAK activity in the brains of patients with Alzheimer's disease (AD) and suggest that this might cause the dendritic spine and cognitive defects observed in $\mathrm{AD}$.

PAK signalling inactivates cofilin, which destabilizes interactions between actin subunits, causing it to detach from actin. This enables another protein, drebrin, to bind and regulate actin in postsynaptic spines. In the absence of cofilin inactivation, pathological rods or inclusions form, features that are characteristic of AD. Zhao et al. show that soluble PAK levels are significantly reduced in the brains of patients with $\mathrm{AD}$, and that phosphorylated PAK is redistributed to granular and tangle-like accumulations, suggesting a link between loss of PAK activity and cofilin aggrega- tion, drebrin loss and synaptic defects observed in $\mathrm{AD}$.

These same features were observed in an Alzheimer's model mouse engineered to produce high levels of amyloid- $\beta(A \beta)$. Reducing the $A \beta$ burden of these mice, by passive immunization with an anti-A $\beta$ antibody, led to an increase in PAK and drebrin levels. Furthermore, the addition of $A \beta$ oligomers to cultured primary hippocampal neurons induced rapid and persistant reductions in PAK and drebrin, which supports a role for $A \beta$ in inducing PAK signalling defects. Expressing wild-type PAK1 in these neurons limited drebrin loss; conversely, pharmacological inhibition of PAK in adult mice recapitulated many of the features of $\mathrm{AD}$.

These findings suggest that PAK and its downstream effectors represent potential therapeutic targets for AD.

$$
\text { Daniel McGowan }
$$

ORIGINAL RESEARCH PAPER Zhao, L. et al. Role of p21-activated kinase pathway defects in the cognitive deficits of Alzheimer disease. Nature Neurosci. 9, 234-242 (2006)

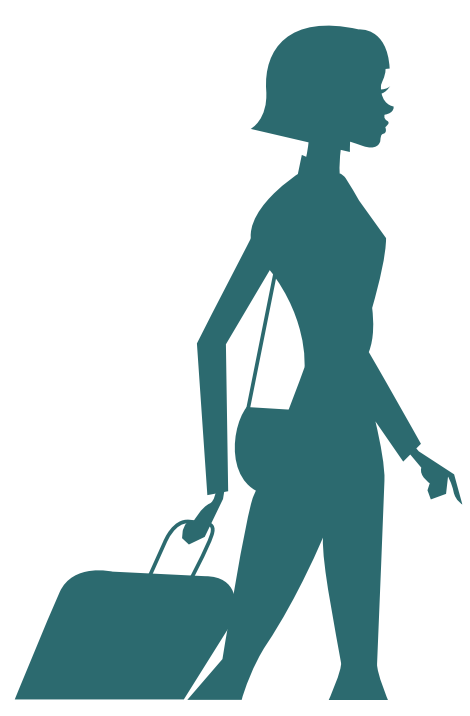

